\title{
Fluid Flow and Heat Transfer in an Internal Coolant Passage
}

\author{
TOM I.-P. SHIH ${ }^{\mathrm{a}, *}$, YU-LIANG LIN ${ }^{\mathrm{a}}$ and MARK A. STEPHENS ${ }^{\mathrm{b}}$ \\ a Department of Mechanical Engineering, Michigan State University, East Lansing, Michigan 48824-1226 USA; \\ ${ }^{\mathrm{b}}$ Department of Mechanical Engineering, Carnegie Mellon University, Pittsburgh, Pennsylvania 15213-3890 USA
}

(Received 22 March 1999; In final form 15 June 2000)

\begin{abstract}
Computations were performed to study the three-dimensional flow and heat transfer in a $U$-shaped duct of square cross section with inclined ribs on two opposite walls under rotating and non-rotating conditions. Two extreme limits in the Reynolds number $(25,000$ and 350,000$)$ were investigated. The rotation numbers investigated are $0,0.24$, and 0.039 . Results show rotation and the bend to reinforce secondary flows that align with it and to retard those that do not. Rotation was found to affect significantly the flow and heat transfer in the bend even at a very high Reynolds number of 350,000 and a very low Rotation number of 0:039. When there is no rotation, the flow and heat transfer in the bend were dominated by rib-induced secondary flows at the high Reynolds number limit and by bend-induced pressure-gradients at the low Reynolds number limit. Long streaks of reduced surface heat transfer occur in the bend at locations where streamlines from two contiguous secondary flows merge and then flow away from the surface. The location and size of these streaks varied markedly with Reynolds and rotation numbers.

This computational study is based on the ensemble-averaged conservation equations of mass, momentum (compressible Navier-Stokes), and energy. Turbulence is modelled by the low-Reynolds shear-stress transport (SST) model of Menter. Solutions were generated by using a cell-centered, finite-volume method, that is based on second-order accurate flux-difference splitting and a diagonalized alternating-direction implicit scheme with local time-stepping and $V$-cycle multigrid.
\end{abstract}

Keywords: Rotating duct; Curved duct; Turbine cooling

\section{INTRODUCTION}

To increase thermal efficiency and specific thrust, advanced gas turbine stages are being designed to operate at gas temperatures that exceed acceptable material temperatures. Even with the development of thermal-barrier coatings, cooling is needed for all surfaces that come in contact with the hot gases, especially in the first stage.

\footnotetext{
*Corresponding author. Tel.: 517 432-3658, Fax: 517 353-1750, e-mail: tomshih@egr.msu.edu
} 
One effective and widely used method of cooling is internal cooling. With internal cooling, lowertemperature air is extracted from the compressor and circulated through passages embedded inside components such as blades and vanes. Efficiency considerations demand effective cooling with minimal cooling air. This need for efficiency has led numerous investigators to study fluid flow and heat transfer processes inside internal coolant passages and to develop and evaluate design concepts that enhance heat transfer with minimal drag.

Experimental studies on internal coolant passages have focused mostly on non-rotating ducts, which are relevant to vanes. See, for example, Han et al. (1992); Chyu et al. (1995); Liou et al. (1998), and references cited there. Experimental studies on rotating ducts, which are relevant to blades, have been less numerous. Wagner et al. (1991a, b); Morris and Salemi (1992); Han et al. (1994) and Cheah et al. (1996) investigated rotating ducts with smooth walls. Taslim et al. (1991); Wagner et al. (1992); Zhang et al. (1993); Johnson et al. (1994); Zhang et al. (1995); Tse (1995) and Kuo and Hwang (1996) reported studies on rotating ducts with ribbed walls.

Computational studies of internal coolant passages that account for the three-dimensional (3-D) nature of the flow and heat transfer have only recently been reported. 3-D studies are necessary if there are ribs, $180^{\circ}$ bends, and/or rotation. Besserman and Tanrikut (1991); Wang and Chyu (1994) and Rigby et al. (1996) studied non-rotating smooth ducts with $180^{\circ}$ bends. Iacovides et al. (1991, 1996); Medwell et al. (1991); Tekriwal (1991); Dutta et al. (1994); Tolpaldi (1994); Stephens et al. (1996a) and Hwang et al. (1997) studied rotating smooth ducts. Prakash and Zerkle (1992, 1995); Abuaf and Kercher (1994); Stephens et al. (1995a); Rigby et al. (1997) and Rigby (1998) studied ducts with normal ribs.

Very few investigators performed computational studies on ducts with inclined ribs, which are the ones used in advanced designs. Stephens et al. (1995b, 1996b) studied inclined ribs in a straight duct under non-rotating conditions. Bonhoff et al. (1996) studied inclined ribs in a non-rotating $U$ duct (i.e., a duct with two straight sections and a $180^{\circ}$ bend). More recently, Stephens and Shih (1997); Bonhoff et al. (1997) and Shih et al. (1998) studied inclined ribs in $U$-ducts under rotating conditions.

Of the computational studies, only Shih et al. (1998) studied the flow and heat transfer in a ribbed $U$-duct under typical engine conditions (i.e., not only are the geometry, Reynolds number, and Rotation number typical, but also the engine speed, the coolant temperature and pressure, and the wall temperatures). They focused on industrial gas turbines and investigated an extreme situation in which the Reynolds number is 350,000 under both rotating and non-rotating conditions. When there is rotation, the duct rotated at $3,600 \mathrm{rpm}$. They compared their results for a high Reynoldsnumber limit with that of Stephens and Shih (1997) for a low Reynolds-number limit at 25,000. However, that comparison was incomplete for two reasons. First, Stephens and Shih (1997) only had results for the rotating case but not for the nonrotating case. Second, only results on secondary flow and surface heat transfer were given. The objective of this study is to present a more complete investigation of the flow and heat transfer in a ribbed $U$-duct involving Reynolds numbers at two extreme limits under both rotating and non-rotating conditions.

\section{DESCRIPTION OF PROBLEM}

A schematic diagram of the problem investigated in this study is shown in Figure 1. It involves flow in a $U$-shaped duct of square cross section, made up of two straight sections and a $180^{\circ}$ bend. The geometry of the straight section is the same as that reported in Wagner et al. (1991a, b). The geometry of the bend is somewhat different, and is taken from the current experimental setup at United Technologies Research Center (Wagner and Steuber, 1994). The dimensions of the duct are as 


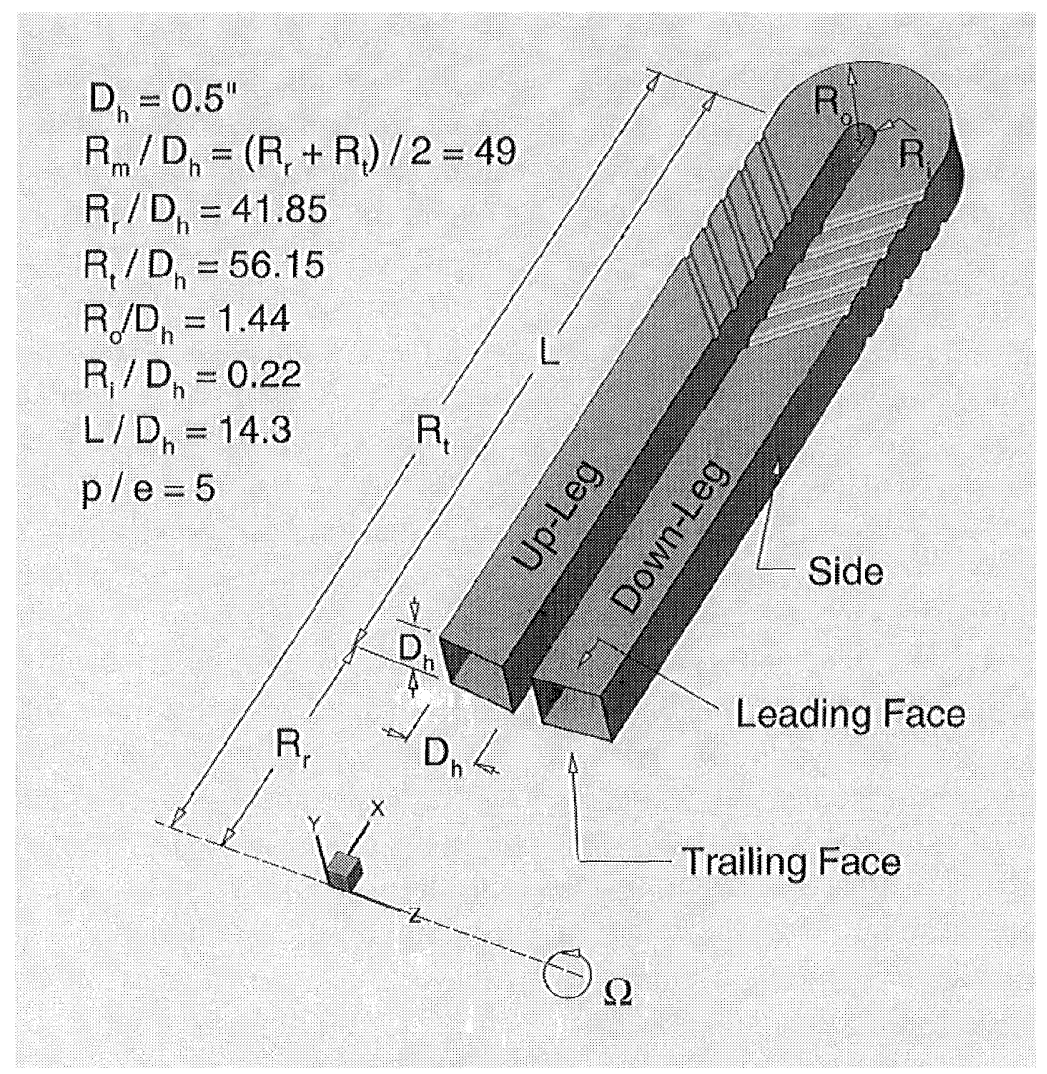

FIGURE 1 Schematic of ribbed $U$-duct studied.

follows (see Fig. 1). The duct hydraulic diameter is $D_{h}=1.27 \mathrm{~cm}(0.5 \mathrm{in})$. The radial position of the duct relative to the axis of rotation is given by $R_{r} / D_{h}=41.85$ and $R_{t} / D_{h}=56.15$ so that the mean radius is $R_{m} / D_{h}=\left(R_{r}+R_{t}\right) / 2 D_{h}=49$. The length of the duct's straight section is $L / D_{h}=14.3$. The curvature of the $180^{\circ}$ bend is given by $R_{i} / D_{h}=0.22$ and $R_{o} / D_{h}=1.44$.

The leading and trailing walls of the straight sections are each roughened with five equally spaced ribs. The ribs on those two walls are staggered relative to each other with ribs on the leading wall offset upstream from those on the trailing wall by a half pitch $(p)$. The ribs are located just upstream or downstream of the $180^{\circ}$ bend. All ribs are inclined with respect to the flow at an angle of $45^{\circ}$. The cross section of the rounded ribs is made up of three circular arcs of radius $0.0635 \mathrm{~cm}(0.025 \mathrm{in})$ so that the rib height (e) is $0.127 \mathrm{~cm}(0.05 \mathrm{in})$ and the rib-height to hydraulic-diameter $\left(e / D_{h}\right)$ is 0.1 . The pitch-toheight ratio $(p / e)$ was set to 5 (same as UTRC experiments). With only five ribs on each face, a considerable length of the duct has smooth walls. See Stephens and Shih (1997) for details on the ribs.

For this problem, four different cases were studied, representing different limits in Reynolds and rotation numbers. A summary of all cases studied is given in Table I. For Cases 1 and 2, the four walls of the duct including the rib surfaces were maintained at a constant temperature of $T_{w}=800 \mathrm{~K}$. The coolant air entering the duct had a uniform temperature of $T_{i}=550 \mathrm{~K}$ and an average static pressure of $P_{i}=10 \mathrm{~atm}$. The Reynolds number at the inlet was $R e=350,000$. 
TABLE I Summary of cases studied*

\begin{tabular}{lcccl}
\hline Case \# & $R e$ & $\Omega(\mathrm{rpm}) / R o$ & $T_{i}(\mathrm{~K})$ & $T_{w}(\mathrm{~K})$ \\
\hline 1 & 350,000 & $0 / 0$ & 550 & 800 \\
2 & 350,000 & $3,600 / 0.039$ & 550 & 800 \\
3 & 25,000 & $0 / 0$ & 300 & 344.83 \\
4 & 25,000 & $3,132 / 0.24$ & 300 & 344.83 \\
\hline
\end{tabular}

*For Cases 1 and $2, P_{i}=10 \mathrm{~atm}, M_{i}=0.26$ and $\Delta \rho / \rho=0.3125$. For Cases 3 and $4, P_{i}=1.78$ atm, $M_{i}=0.05$ and $\Delta \rho / \rho=0.13$.

The angular speed of the rotating duct was $3,600 \mathrm{rpm}$ (376.99 radians per second). These conditions correspond to a rotation number of $R o=0.039$; an inlet Mach number (relative to duct) of $M_{i}=0.26$; and an inlet density ratio of $\Delta \rho / \rho=0.3125$. For Cases 3 and 4 , which represent another limit, the duct wall temperature was $T_{w}=344.83 \mathrm{~K}$, and the coolant temperature, pressure, and Reynolds number at the inlet were $T_{i}=300 \mathrm{~K}, P_{i}=1.78 \mathrm{~atm}$, and $R e=25,000$. These gave rise to an inlet Mach number of $M_{i}=0.05$ and an inlet density ratio of $\Delta \rho / \rho=0.13$. When there is rotation, the angular speed was $3,132 \mathrm{rpm}$, giving rise to a rotation number of $R o=0.24$.

Unlike the temperature profile, the velocity profile at the inlet was not uniform because of the extensive flow passages upstream of it. Since fully developed velocity profiles do not exist for compressible flows, the velocity profile used is the one at the exit of a non-rotating straight duct of length $150 D_{h}$ with adiabatic walls and the same cross section and flow conditions as the $U$-duct studied here.

Of the four cases studied, Cases 1 and 2 are fairly typical of industrial gas turbines except that the Reynolds number is near the upper limit. Cases 3 and 4 are less typical in terms of the pressure and temperature conditions, but the rotation number is fairly typical of the lower Reynolds number limit. Note that for industrial gas turbines, the engine speed is either 3,000 or $3,600 \mathrm{rpm}$ so that for a given geometry and inlet conditions, the higher the Reynolds number, the lower is the rotation number and vice versa.

\section{PROBLEM FORMULATION}

The problem described in the above section and depicted in Figure 1 is modeled by the ensembleaveraged conservation equations of mass (continuity), momentum (compressible NavierStokes), and total energy for a thermally and calorically perfect gas with Sutherland's model for dynamic viscosity and a constant Prandtl number. These equations are written in a coordinate system that rotates with the duct so that steady-state solutions with respect to the duct can be computed. The form of these equations is well known (see, e.g., Steinthorsson et al., 1991; Prakash and Zerkle, 1995 and Stephens and Shih, 1999). In this study, the ensemble-averaged equations were closed by a low-Reynolds-number two-equation $k-\omega$ model known as the shear-stress-transport (SST) model (Menter, 1993 and Menter and Rumsey, 1994). The SST model was selected over the standard $k-\omega$ model of Wilcox (1993) because it eliminates dependence on freestream $k$ and $\omega$, and has a limiter to control overshoot in $k$ with adverse pressure gradients so that separation is predicted more accurately.

Note that by being a low-Reynolds number model, integration of the conservation equations and the turbulence model is made all the way to the wall. Thus, the wall boundary conditions (BCs) are zero velocity, constant wall temperature and zero turbulent kinetic energy. The dissipation rate per $k(\omega)$ on the wall was set to $100(\partial u / \partial n)_{w}$ as proposed by Wilcox (1993) for hydraulically smooth surfaces. A check, however, was imposed to ensure that the maximum value of $\omega$ was less than $60 \nu /\left(\beta \operatorname{Re} \Delta y^{2}\right)$, where $\Delta y$ is the normal distance from the wall and $\beta=3 / 40$, as suggested by Menter (1993). Other BCs are as follows. At the duct entrance, a developed profile was specified for velocity, but the temperature profile was taken to be uniform (see previous section for details). Turbulence quantities ( $k$ and $\omega$ ) were specified in a manner that is consistent with the velocity profile (average turbulent intensity was $5 \%$ ). Only pressure was extrapolated. At the duct 
exit, an average static pressure was imposed but the pressure gradients in the two spanwise directions were extrapolated. This is important because secondary flows induced by inclined ribs, the bend, and centripetal/Coriolis forces cause considerable pressure variations in the spanwise directions. Density and velocity were also extrapolated.

Though only steady-state solutions are of interest in this study, initial conditions were needed because the unsteady form of the conservation equations was used. The initial conditions used are the solutions of the steady, one-dimensional, inviscid equations, namely,

$$
\frac{\partial \rho u}{\partial x}=0, \frac{\partial \rho u^{2}}{\partial x}=-\frac{\partial P}{\partial x}+\rho \Omega^{2} x, \frac{\partial \rho u \hbar}{\partial x}=\rho u \Omega^{2} x
$$

where $\hbar$ is enthalpy. For an ideal gas, the solution to Eq. (1) is

$$
\begin{aligned}
\frac{T}{T_{i}} & =1+\frac{\Omega^{2}}{C_{p} T_{i}} \frac{x^{2}-R_{r}^{2}}{2} \\
\frac{T}{T_{i}} & =\left(\frac{\rho}{\rho_{i}}\right)^{(\gamma-1)}=\left(\frac{P}{P_{i}}\right)^{((\gamma-1) / \gamma)} \\
& =\left(\frac{u_{i}}{u}\right)^{(1 /(\gamma-1))}
\end{aligned}
$$

\section{NUMERICAL METHOD OF SOLUTION}

Solutions to the ensemble-averaged conservation equations were obtained by using a cell-centered finite-volume code called CFL3D (Thomas et al., 1990 and Rumsey and Vatsa, 1993). In this study, the CFL3D code was modified so that it can account for steady-state solutions in a rotating frame of reference by adding source terms that represent centripetal and Coriolis forces in the momentum and energy equations.

The CFL3D code contains a number of different algorithms. The one used in this study is as follows. All inviscid terms were approximated by the second-order accurate flux-difference splitting of Roe (1981). All diffusion terms were approximated conservatively by differencing derivatives at cell faces. Since only steady-state solutions were of interest, time derivatives were approximated by the Euler implicit formula. The system of nonlinear equations that resulted from the aforementioned approximations to the space- and time-derivatives were analyzed by using a diagonalized alternatingdirection implicit scheme (Pulliam and Chaussee, 1981) with local time-stepping (local Courant number always set to unity) and three-level $V$ cycle multigrid (Anderson et al., 1988).

The domain of the problem was replaced by a single-block grid system that was assembled by patching together 37 blocks of $\mathrm{H}-\mathrm{H}$ structured grids with $C^{2}$ continuity at all block interfaces (see Fig. 2). For this grid system, the total number of grid points in the streamwise direction from inflow to outflow is 761 . The number of grid points in the cross-stream plane is $65 \times 65$. From Figure 2, it can be seen that grid lines are clustered near all solid surfaces to resolve the sharp gradients there, are smooth as grid spacings change from fine to coarser, and are orthogonal everywhere except along rib surfaces. Along rib surfaces, the grid lines are aligned with the rib inclination. The grid system used satisfy a set of rules that are needed to obtain meaningful solutions such as having five grid points within $y^{+}$of 5 . The rules and the process can be found in Stephens et al. (1996a), and will not be repeated here. As a further test, the aforementioned grid systems were refined by a factor of $25 \%$, first in the streamwise and then in the cross-stream directions. This grid independence study showed the predicted surface heat transfer coefficient to vary by less than $2 \%$.

On a Cray C-90 computer, where all computations were performed, the memory requirement for each run is 155 megawords (MWs). The CPU time required for a converged steady-state solution is about 40 hours and involves about 3,000 iterations. A solution is said to be converged when the following conditions are satisfied: (1) residual drops at least three orders of magnitude; (2) the second norm of the residual is less than $10^{-13}$; and 


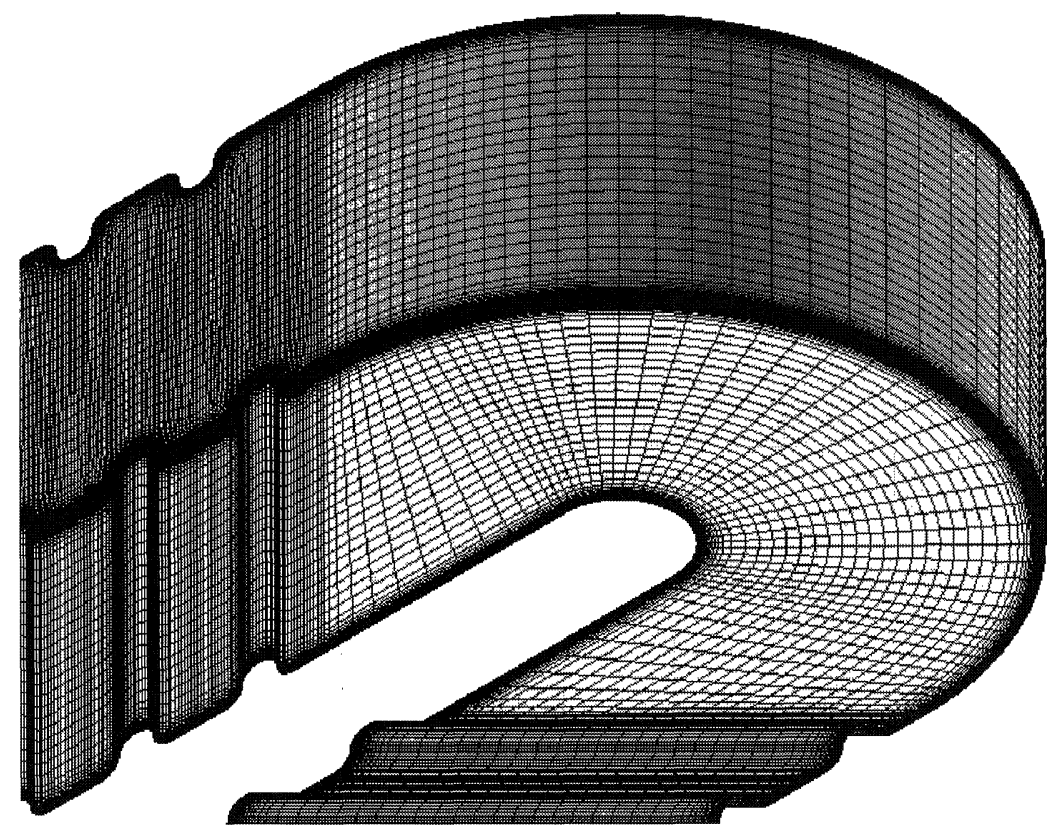

FIGURE 2 Grid system used.

(3) predicted surface heat transfer varies by less than $1 \%$.

The code used in this study has been validated for two problems. The first problem is flow in a non-rotating square duct in which two opposite walls are lined with inclined ribs. In that problem, the geometry of the duct and rib is identical to that studied here for the up-leg portion of the $U$ duct. The computed heat-transfer enhancement for that problem was compared with measurements obtained by using liquid crystal thermography (see Stephens et al., 1996a). The comparisons showed that the code used predicted the qualitative features very well. The predicted quantitative features were also found to be reasonable (peak values are within 10 to $20 \%$ ). The second problem is flow in a rotating $U$-duct with smooth walls. The geometry of that problem is the same as that studied here except for the absence of ribs. For that problem, the computed Nusselt numbers on the leading and trailing walls for the up-leg part of the $U$-duct were compared with measured one by Wagner et al. (1991a). Those comparisons, described in Stephens et al. (1996b), showed very good agreements. The good comparisons obtained in those two studies give some confidence to the code for studying the present problem.

\section{RESULTS}

As noted in the Introduction, the objective of this study is to investigate the flow and heat transfer in a ribbed $U$-duct for two extremes in Reynolds number under rotating and non-rotating conditions. To meet this objective, computations were performed for four cases summarized in Table I. The results generated are given in Figures 3 to 6 .

\section{Flow Field}

The flow field in the $U$-duct with ribs and a coolant-to-wall temperature that is less than unity can be inferred from Figures 3 to 5 . Figures 3 and 4 show projected velocity vector fields colored by 


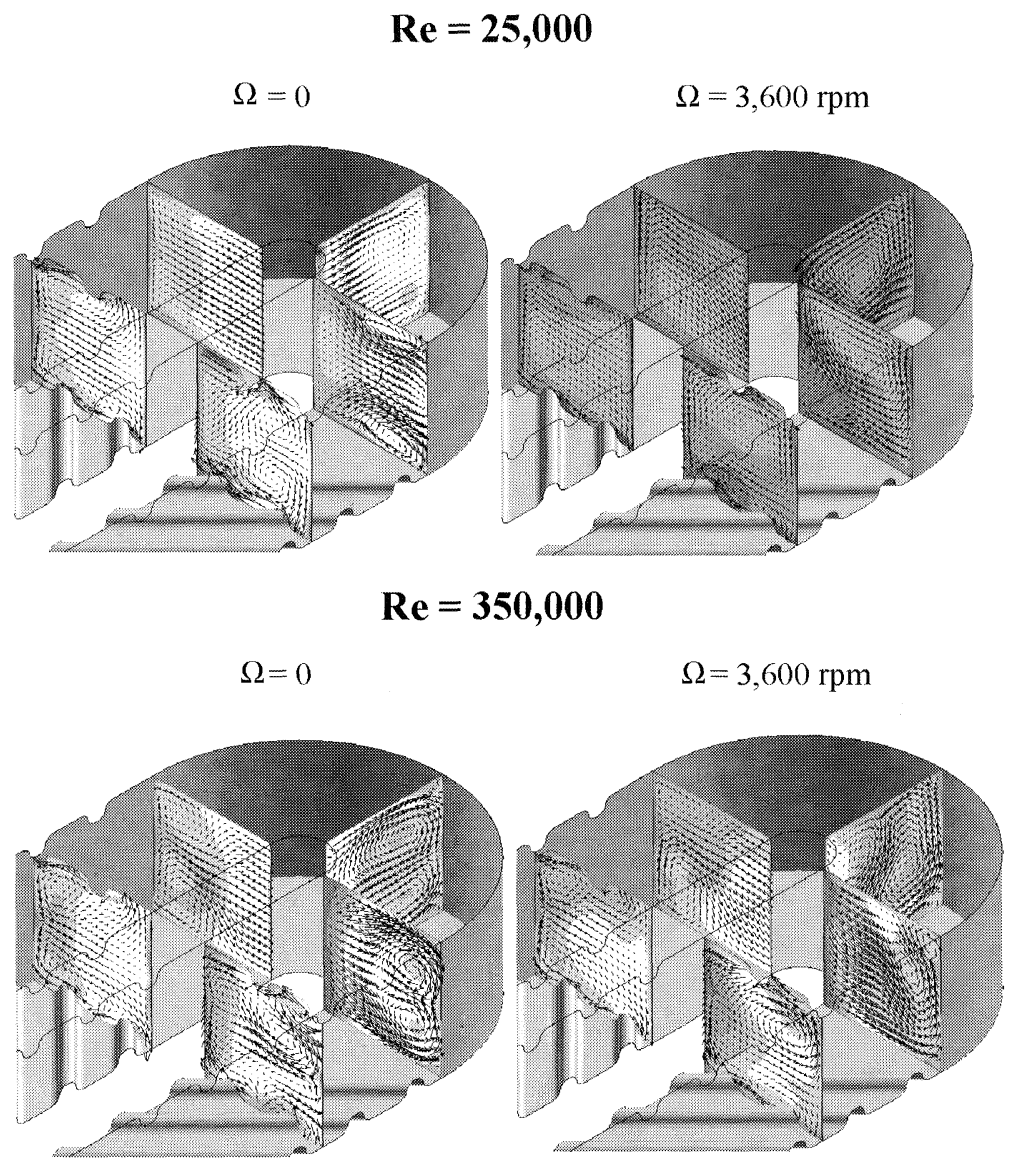

FIGURE 3 Normalized temperature with projected velocities at several cross-stream planes.

normalized temperature, $\eta$, along several planes. Figure 5 shows normalized pressure, $P / P_{\text {ref }}$, on walls with velocity vectors at $0.0016 D_{h}$ from walls. Here, the coloration is in gray scale, which can be difficult to read. Interested reader can contact the first author to obtain colored plots.

Before discussing the results in these figures, it is important to review the nature of the flow in a smooth $U$-duct under non-rotating and rotating conditions. When there is no rotation $(R o=0.0$, $R e=25,000)$, Stephens et al. (1996b) and Lin et al. (1998) showed that the velocity profile to have a maximum about the center of the ductcross-section in the up-leg portion. As the flow approaches the bend, it is decelerated near the concave part of the bend with some flow reversal near the leading/trailing walls because of the adverse pressure gradient induced by the bend. Near the convex part of the bend, the flow is accelerated instead of decelerated. As the flow goes around the bend, Dean-type secondary flows form, and these secondary flows persist in the down-leg part of duct. Around the bend, a large streamwise separation region forms on the convex side of the bend. The Dean-type secondary flows induce another pair of secondary flows in the separated region downstream of the bend.

When there is rotation $(R o=0.24, R e=25,000)$, Stephens and Shih (1997, 1999); Lin et al. (1998) and Shih et al. (1999) showed that a pair of counter-rotating flows form in the up-leg part of duct because of the Coriolis force. With radially 

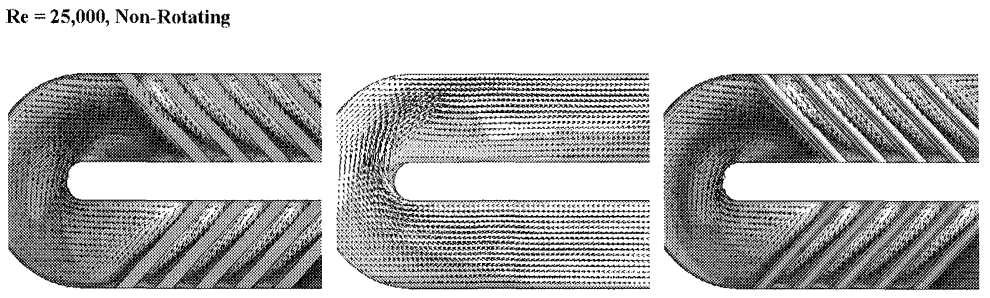

$\operatorname{Re}=25,000$, Rotating
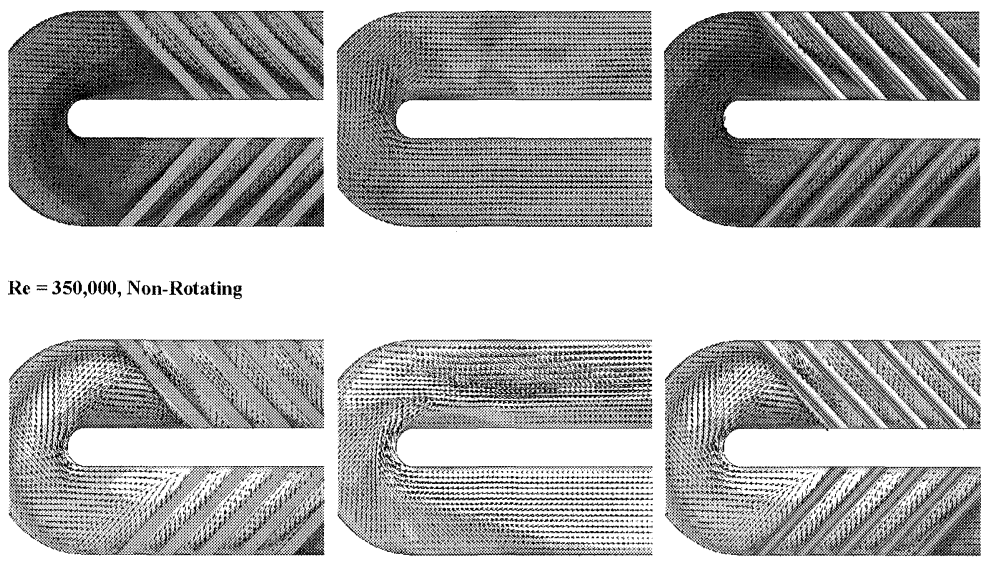

$\operatorname{Re}=350,000$, Rotating
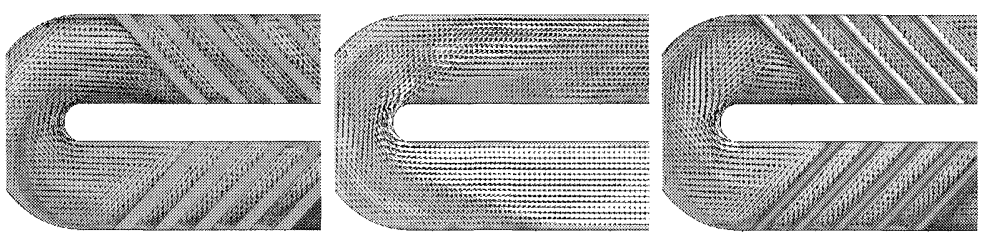

FIGURE 4 Normalized temperature with projected velocities at several streamwise planes. Left: leading, Middle: mid-plane, Right: trailing. Scale is same as that in Figure 3.

outward flow, this pair flows from the trailing face to the leading face along the two side walls, which transports cooler air from near the center of the duct cross section to the trailing wall. Since the thermal boundary layer starts on the trailing wall, air temperature near the trailing wall is lower than that near the leading wall. With higher temperature and hence lower density near the leading wall, centrifugal buoyancy decelerates the flow there. With lower velocity and hence thicker boundary layer next to the leading face, the Coriolis-induced secondary flows cause the formation of additional pairs of vortices near the leading face (Stephens and Shih, 1999). With the above as a backdrop, the results of this study given in Figures 3 to 5 are described.

\section{Non-rotating Ribbed Duct}

When there is no rotation (Cases 1 and 3, Tab. I), the flow in the $U$-duct is dominated by the inclined ribs. The staggered inclined ribs induce two counter-rotating secondary flows with the sense of rotation dependent upon the rib inclination. In the 
up-leg part, the secondary flows flow from the inner side wall to the outer side wall along the two faces of the duct with ribs. Note that there is flow reversal in the region between the ribs (see Figs. 3 and 4). Because of the ribs' staggered arrangement, the size of these secondary flows oscillates along the duct. The magnitude of this oscillation is weaker at the higher Reynolds number. These secondary flows transport cooler fluid towards the ribbed faces.

As the flow approaches the bend, the secondary flow components have higher values near the concave wall than the convex wall (Fig. 4). This is because the flow component along the duct is decelerated near the concave wall and accelerated near the convex wall. Further into the bend, the pressure gradient induced by the flow curvature reinforces secondary flows that are aligned with
Dean-type ones and retards those that do not (Fig. 3). At $R e=25,000$, the secondary flow in the bend is asymmetric with bend-induced pressure gradient exerting considerable influence. At $R e=$ 350,000 , however, the secondary flows in the bend are nearly symmetric with the effects of the bend-induced pressure gradient being minimal. Note that whenever different secondary flows meet near surfaces, the temperature reaches a local maximum.

Around the bend, there is a large separated region next to the convex surface. This separated region extends all the way to the first rib in the down-leg part of the $U$-duct. The size of the separation bubble is larger when $R e$ is lower. Unlike smooth $U$-ducts, there was essentially no flow separation next to the concave wall of the bend.
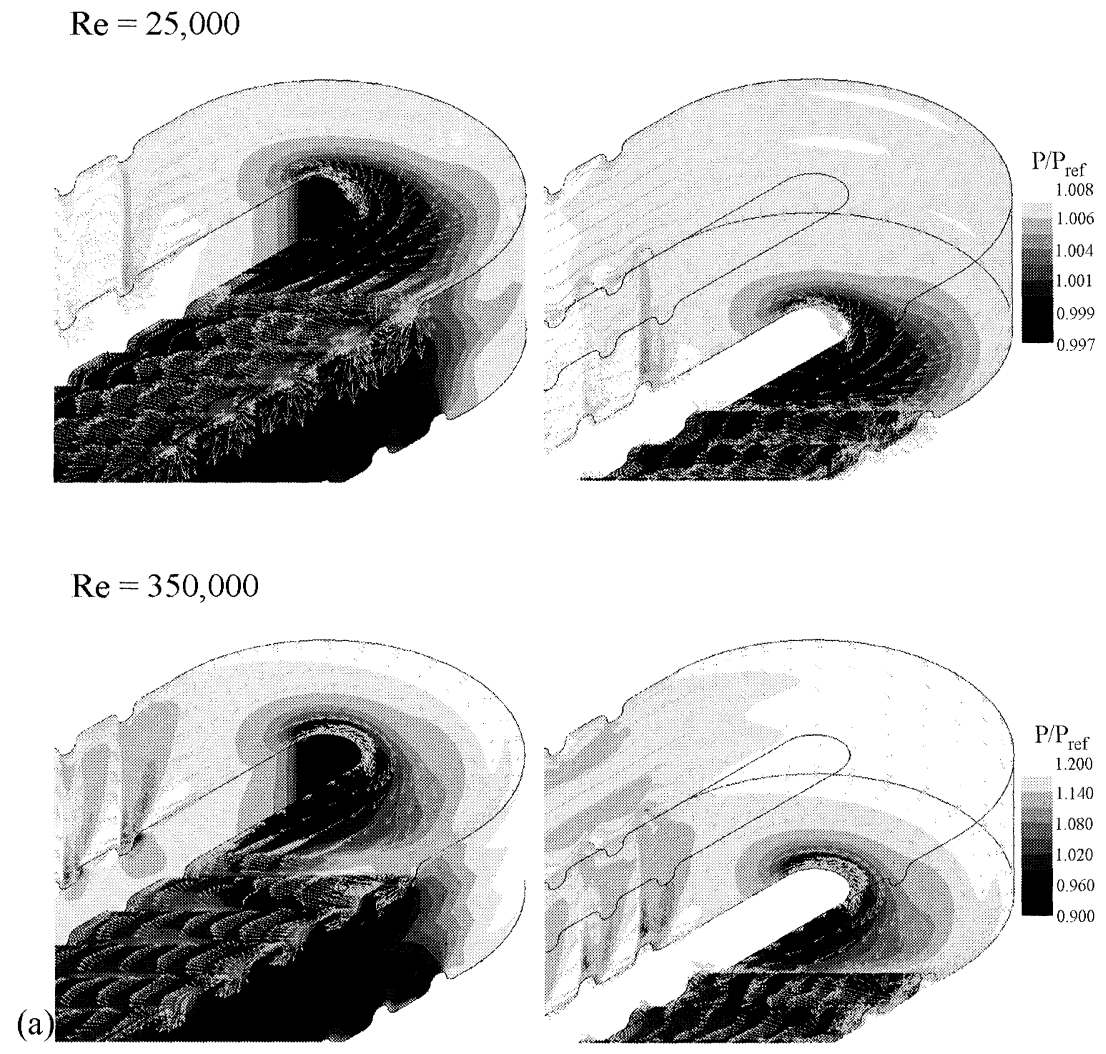

FIGURE 5 Normalized pressure on walls with velocity vectors at $0.0016 D_{h}$ from walls. Left: leading, Right: trailing, (a) Nonrotating; (b) Rotating. 


$$
\operatorname{Re}=25,000
$$

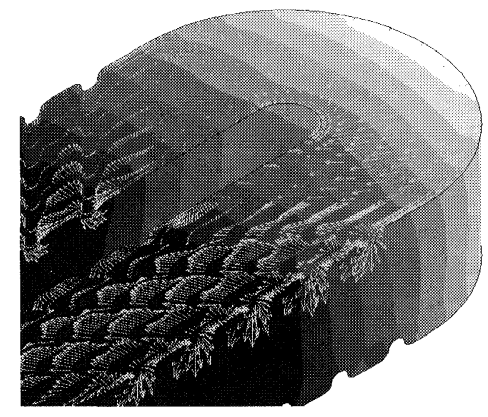

$$
\operatorname{Re}=350,000
$$

(b)

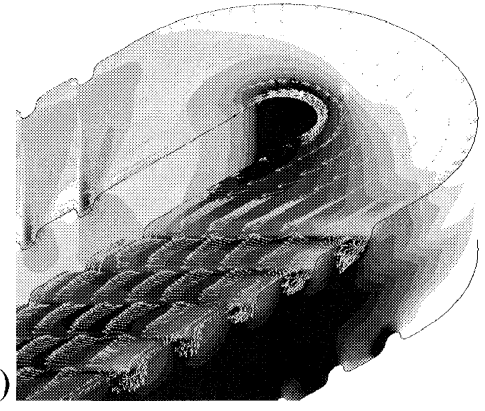

FIGURE 5

If the rib inclination was $-45^{\circ}$ instead of $+45^{\circ}$ in the up-leg part of the duct, then Dean-type secondary flows in the bend would reinforce ribinduced secondary flows. To continue this reinforcement in the down-leg part of the duct, the rib inclination there should remain at $+45^{\circ}$.

\section{Rotating Ribbed Duct}

With rotation (Cases 2 and 4, Tab. I), Coriolisinduced secondary flows in the smooth part of duct are quickly dominated by those induced by inclined ribs. However, at $R e=25,000$, Coriolis force can clearly be seen to reinforce the secondary flow that is aligned with it (i.e., turning in the same direction) and to retard the one that is opposed to it. Thus, one secondary flow (the one next to the leading face) becomes larger and stronger than the other. At $R e=350,000$, the effects of Coriolis diminishes but its effects are clearly not negligible
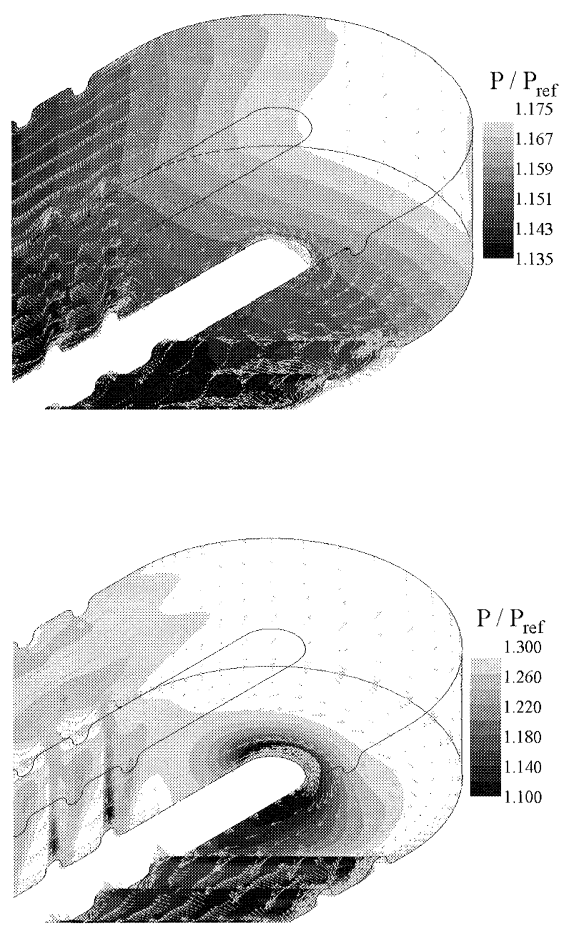

(Continued).

by noting differences in the flow with and without rotation.

Around the bend, the curvature-induced pressure gradient further reinforces the secondary flow that is aligned with it and retards the one that is not. At $R e=25,000$, one secondary flow dominate the entire duct cross section. At $R e=350,000$, the two secondary flows created by inclined ribs in the up-leg part persist throughout the bend, but they are definitely affected by the pressure gradient there by becoming highly asymmetric (Fig. 3).

When there is rotation, flow reversal occurs in the bend next to the leading and trailing faces when $R e=25,000$, but not when $R e=350,000$. Recall that when there is no rotation, flow reversal does not take place if ribs are present at both Reynolds numbers investigated.

In the down-leg part of the $U$-duct, the secondary flows are again reinforced or retarded by the Coriolis force, which further accentuate the 
asymmetry created in the up-leg part and the bend. Thus, rotation clearly has strong effects on the nature of the flow even at $R e=350,000$ and $R o=0.039$.

\section{Surface Heat Transfer}

The heat transfer characteristics can be inferred from Figures 3, 4 and 6. Figures 3 and 4 show normalized temperature contours, $\eta$, at selected planes, and Figure 6 shows surface heat transfer coefficient via a normalized Nusselt number, $\mathrm{Nu}$ / $N u_{s}$. From these figures, it is noted that whether there is rotation or not, high heat transfer rates occur at the ribs. Low heat transfer rates take place at the following locations on the ribbed faces: just downstream of the rib tops and towards the outer wall for the up-leg part; just downstream of the rib tops and towards the inner wall for the down-leg part; in the separated region just downstream of the bend; and in thin strips on the leading and trailing faces in the bend where different secondary flow leave the surface. Basically, when two secondary flows impinge on a surface, heat transfer is high, and when they leave a surface, heat transfer is low. All streaks of low and high surface heat transfer in the bend can be attributed to this. Thus, the structure of the secondary flows in the bend has a significant effect on surface heat transfer.

When there is no rotation, the heat-transfer enhancement on the ribbed faces is nearly identical with the average heat transfer rate in the up-leg part higher than that in the down-leg part. In the bend, the effects of increasing $R e$ from 25,000 to 350,000 is to shift the streak of low heat-transfer

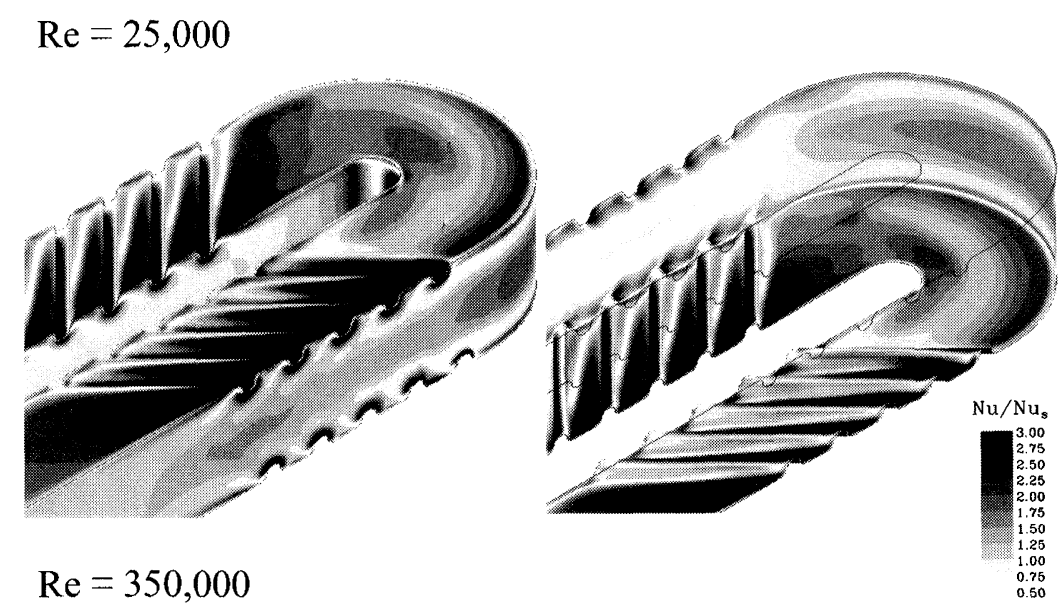

(a)

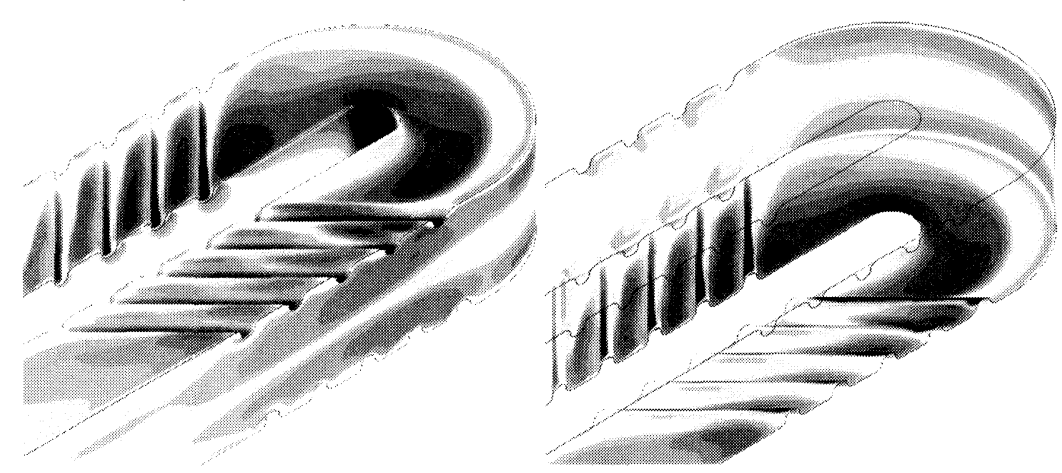

FIGURE $6 N u / N u_{s}$ on the leading, trailing, and side walls. Left: leading, Right: trailing, (a) Non-rotating; (b) Rotating. 


$$
\operatorname{Re}=25,000
$$

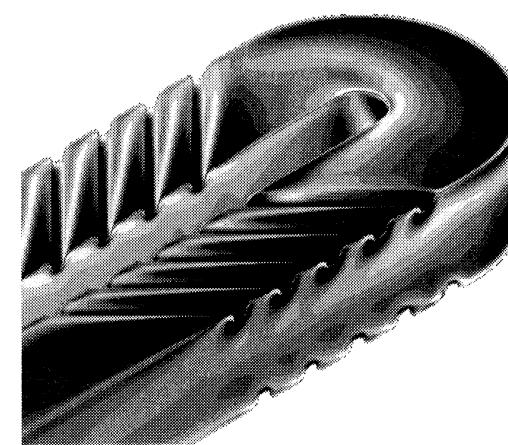

$\operatorname{Re}=350,000$

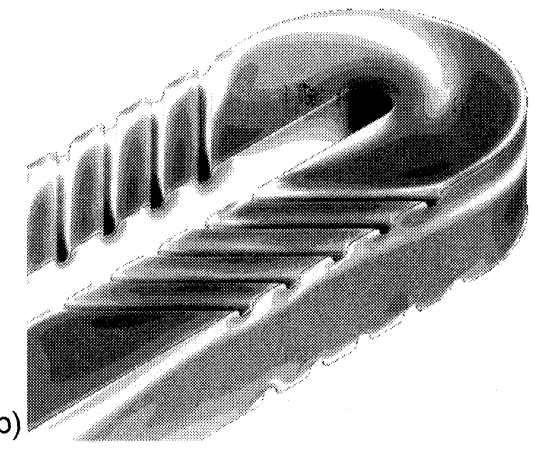

FIGURE 6 (Continued).

enhancement from near the middle of the leading and trailing faces towards the concave wall. On the concave wall, a similar increase in $R e$ causes the two streaks of low heat-transfer enhancement to be near its middle instead of near the ribbed walls.

When there is rotation, the heat-transfer enhancement on the trailing face is markedly higher than that on the leading face in the up-leg part. In the down-leg part, heat-transfer enhancement is higher on the trailing wall when $R e=350,000$ and higher on the leading wall when $R e=25,000$. The reason is that hotter fluid was convected to the trailing wall at $R e=25,000$. Again, secondary flows affect surface heat transfer by transporting hotter or cooler fluid to the leading and trailing faces.
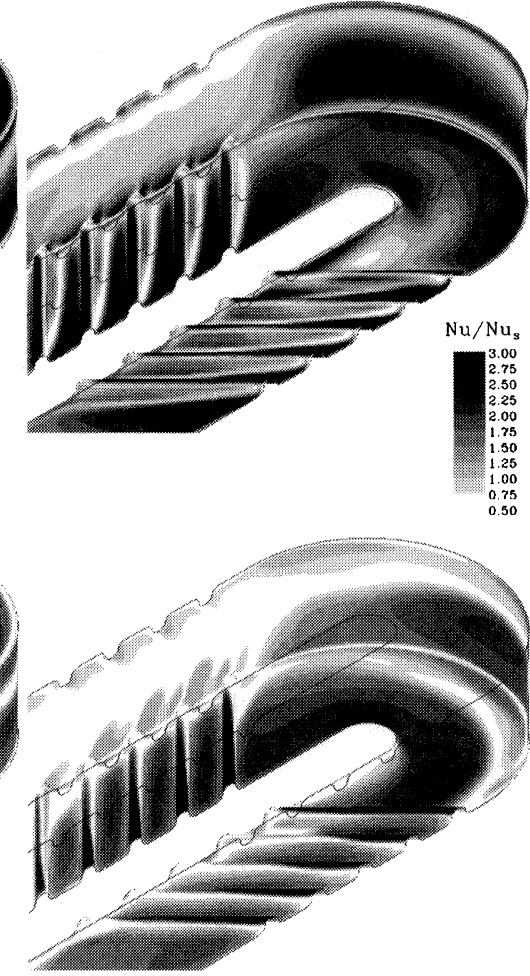

Computations were performed by using a validated CFD code to examine the flow and heat transfer in a ribbed $U$-shaped duct with and without rotation. The Reynolds numbers investigated are 25,000 and 350,000, which represent two extreme limits. Results show in detail the evolution of the secondary flows induced by inclined ribs and a smooth 180 bend under rotating and nonrotating conditions. Both rotation and the bend were found to reinforce secondary flows that align with it and to retard those that do not. Even at $R e=350,000$, the effects of rotation were found to be significant. The structure of the secondary flows is important because it determines how cooler fluid 
in the core is convected to the ribbed or leading and trailing faces. The cooler the fluid is near the surface, the higher is the heat transfer rate. Long streaks of reduce surface heat transfer in the bend were found to be due to secondary flows meeting and then leaving the surface. These streaks were a strong function of the Reynolds and rotation numbers.

\section{Acknowledgements}

The authors are grateful for the computer time provided by NAS and the Pittsburgh Supercomputing Center.

\section{NOMENCLATURE}

$C_{p} \quad$ constant pressure specific heat

$D_{h} \quad$ hydraulic diameter of duct

$h \quad$ heat transfer coefficient $\left(q_{w} /\left(T_{w}-T_{b}\right)\right)$

$M_{i} \quad$ Mach number $\left(V_{i} / \sqrt{\gamma \overline{\mathbf{R}} T_{i}}\right)$

$\mathrm{Nu} \quad$ Nusselt number $\left(h D_{h} / \kappa\right)$

$\mathrm{Nu}_{s} \quad$ Nusselt number for smooth duct $\left(N u_{s}=0.023 \operatorname{Re}^{0.8} \operatorname{Pr}^{0.4}\right)$

$P, P_{\text {ref }} \quad$ static pressure, pressure at inlet

$q_{w} \quad$ heat transfer rate per unit area from duct surface

$R_{i}, R_{o} \quad$ inner and outer radius of $180^{\circ}$ bend (Fig. 1)

$R_{r}, R_{t} \quad$ radius from axis of rotation (Fig. 1)

$\overline{\mathrm{R}} \quad$ gas constant for air

Re Reynolds number $\left(\rho_{i} V_{i} D_{h} / \mu\right)$

Ro $\quad$ rotation number $\left(\Omega D_{h} / V_{i}\right)$

$T, T_{i}, T_{w} \quad$ temperature, coolant inlet temp., wall temp.

$T_{b} \quad$ bulk temperature $\left(T_{b}=T_{i}+\left(\Omega^{2} / C_{p}\right)\right.$ $\left.\left(\left(x^{2}-R_{r}^{2}\right) / 2\right)\right)$

$u, v, w \quad x$ -,$y$-, $z$-component velocity relative to duct ( $x$ is along duct)

$V_{i} \quad$ average velocity at duct inlet

$x, y, z \quad$ coordinate system rotating with duct (Fig. 1)

\section{Greek Letters}

$\eta \quad$ normalized temperature, $\left(T-T_{i}\right)$

$\left(T_{w}-T_{i}\right)$

$\rho \quad$ density

$\Delta \rho / \rho \quad\left(T_{w}-T_{i}\right) / T_{w}$

$\gamma \quad$ ratio of specific heats (1.4)

$\kappa \quad$ thermal conductivity

$\mu \quad$ dynamic viscosity

$\Omega \quad$ angular speed of duct

\section{References}

Abuaf, N. and Kercher, D. M. (1994) Heat Transfer and Turbulence in a Turbulated Blade Cooling Circuit, ASME J. of Turbomachinery, 116, 169-177.

Anderson, W. K., Thomas, J. L. and Whitfield, D. L. (1988) Multigrid Acceleration of the Flux-Split Euler Equations, AIAA J., 26(6), 649-654.

Besserman, D. L. and Tanrikut, S. (1991) Comparison of Heat Transfer Measurements with Computations for Turbulent Flow Around a $180^{\circ}$ Bend, ASME Paper 91-GT-2.

Bonhoff, B., Tomm, U. and Johnson, B. V. (1996) Heat Transfer Predictions for $U$-Shaped Coolant Channels with Skewed Ribs and with Smooth Walls, ASME 96-TA-7.

Bonhoff, B., Tomm, U., Johnson, B. V. and Jennions, I. (1997) Heat Transfer Predictions for Rotating $U$-Shaped Coolant Channels with Skewed Ribs and with Smooth Walls, ASME 97-GT-162.

Cheah, S. C., Iacovides, H., Jackson, D. C., Ji, H. and Launder, B. E. (1996) LDA Investigation of the Flow Developmen through Rotating $U$-Ducts, ASME J. of Turbomachinery, 118, $590-596$

Chyu, M. K. and Natarajan, V. (1995) Surface Heat Transfer from a Three-Pass Blade Cooling Passage Simulator, ASME $J$. of Turbomachinery, 117(4), 650-656.

Dutta, S., Andrews, M. J. and Han, J. C. (1994) Simulation of Turbulent Heat Transfer in a Rotating Duct, AIAA J. of Thermophysics and Heat Transfer, 9(2), 381-382.

Han, J. C. and Zhang, Y. M. (1992) High Performance Heat Transfer Ducts with Parallel Broken and $V$-Shaped Broken Ribs, International J. of Heat and Mass Transfer, 35(2), $513-523$

Han, J. C., Zhang, Y. M. and Lee, C. P. (1994) Influence of Surface Heating Condition on Local Heat Transfer in a Rotating Square Channel with Smooth Walls and Radially Outward Flow, ASME J. of Turbomachinery, 116(1), $149-158$

Hwang, J.-J., Wang, W.-J. and Lai, D.-Y. (1997) Numerical Simulation of Turbulent Heat Transfer and Flow in a Rotating Multiple-Pass Square Channel, ASME 97-GT-367.

Iacovides, H. and Launder, B. E. (1991) Parametric and Numerical Study of Fully Developed Flow and Heat Transfer in Rotating Rectangular Ducts, ASME J. of Turbomachinery, 113(3), 331-338.

Iacovides, H., Launder, B. E. and Li, H.-Y. (1996) The Computation of Flow Development through Stationary and 
Rotating $U$-Ducts of Strong Curvature, International $J$. of Heat and Fluid Flow, 17, 22-33.

Johnson, B. V., Wagner, J. H., Steuber, G. D. and Yeh, F. C. (1994) Heat Transfer in Rotating Serpentine Passages with Selected Model Orientations for Smooth or Skewed Trip Walls, ASME Journal of Turbomachinery, 116, 738-744.

Kuo, C. R. and Hwang, G. J. (1996) Experimental Studies and Correlations of Radially Outward and Inward Air-Flow Heat Transfer in a Rotating Square Duct, $A S M E J$. of Heat Transfer, 118(1), 23-30.

Lin, Y.-L., Shih, T. I.-P., Civinskas, K. C., Rigby, D. L. and Ameri, A. A. (1998) Nonlinear Versus Linear $k-\omega$ Mode for Predicting Flow and Heat Transfer in a Smooth $U$-Duct, AIA A Paper 98-3560.

Liou, T.-M., Tzeng, Y.-Y. and Chen, C.-C. (1998) Fluid Flow in a $180^{\circ}$ Sharp Turning Duct with Different Divider Thicknesses, ASME 98-GT-189.

Medwell, J. O., Morris, W. D., Xia, J. Y. and Taylor, C. (1991) An Investigation of Convective Heat Transfer in a Rotating Coolant Channel, ASME J. of Turbomachinery, 113(3), $354-359$.

Menter, F. R. (1991) Performance of Popular Turbulence Models for Attached and Separated Adverse Pressure Gradient Flows, $A I A A J$., 30(8), 2066-2071.

Morris, W. D. and Salemi, R. (1992) An Attempt to Uncouple the Effects of Coriolis and Buoyancy Forces Experimentally on Heat Transfer in Smooth Circular Tubes that Rotate in the Orthogonal Mode, ASME J. of Turbomachinery, 114(4), $858-864$.

Pulliam, W. R. and Chaussee, D. S. (1981) A Diagonal Form of an Implicit Approximate Factorization Algorithm, J. of Computational Physics, 39, 347-363.

Prakash, C. and Zerkle, R. (1992) Prediction of Turbulent Flow and Heat Transfer in a Radially Rotating Square Duct, ASME J. of Turbomachinery, 114(4), 835-846.

Prakash, C. and Zerkle, R. (1995) Prediction of Turbulent Flow and Heat Transfer in a Ribbed Rectangular Duct with and without Rotation, ASME J. of Turbomachinery, 117, $255-264$

Roe, P. L. (1981) Approximate Riemann Solvers, Parameter Vector and Difference Schemes, J. of Computational Physics, 43, 357-72.

Rumsey, C. L. and Vatsa, V. N. (1993) A Comparison of the Predictive Capabilities of Several Turbulence Models using Upwind and Central-Difference Computer Codes, AIAA Paper 93-0192.

Shih, T. I.-P., Lin, Y.-L., Stephens, M. A., Chyu, M. K. and Civinskas, K. C. (1998) Flow and Heat Transfer in a Ribbed $U$-Duct under Typical Engine Conditions, ASME Paper 98-GT-213

Shih, T. I.-P., Lin, Y.-L., Stephens, M. A., Chyu, M. K. and Civinskas, K. C. (1999) Computations of Flow and Heat Transfer in a Smooth and Ribbed $U$-Duct with and without Rotation, AJTE99/6483, Proceedings of the ASME/JSME Joint Thermal Engineering Conference.

Steinthorsson, E., Shih, T. I.-P. and Roelke, R. J. (1991) Computations of the Three-Dimensional Flow and Heat Transfer within a Coolant Passage of a Radial Turbine Blade, AIAA Paper 91-2238.

Stephens, M. A., Shih, T. I.-P. and Civinskas, K. C. (1995a) Computation of Flow and Heat Transfer in a Rectangular Channel with Ribs, AIAA Paper 95-0180.
Stephens, M. A., Shih, T. I.-P. and Civinskas, K. C. (1995b) Effects of Inclined Rounded Ribs on Flow and Heat Transfer in a Square Duct, AIAA Paper 95-2115.

Stephens, M. A., Chyu, M. K., Shih, T. I.-P. and Civinskas, K. C. (1996a) Calculations and Measurements of Heat Transfer in a Square Duct with Inclined Ribs, AIAA Paper 96-3163.

Stephens, M. A., Shih, T. I.-P. and Civinskas, K. C. (1996b) Computations of Flow and Heat Transfer in a Rotating $U$ Shaped Square Duct with Smooth Walls, AIAA Paper 963161

Stephens, M. A. and Shih, T. I.-P. (1997) Computations of Compressible Flow and Heat Transfer in a Rotating Duct with Inclined Ribs and a $180^{\circ}$ Bend, ASME Paper 97-GT192.

Stephens, M. A. and Shih, T. I.-P. (1999) Computations of Flow and Heat Transfer in a Smooth $U$-Shaped Square Duct with and without Rotation, AIAA Journal of Propulsion and Power, 15(2), 272-279.

Taslim, M. E., Rahman, A. and Spring, S. D. (1991) An Experimental Investigation of Heat Transfer Coefficients in a Spanwise Rotating Channel with Two Opposite RibRoughened Walls, ASME J. of Turbomachinery, 113(1), $75-82$.

Tekriwal, P. (1991) Heat Transfer Predictions with Extended $k-\omega$ Turbulence Model in Radial Cooling Ducts Rotating in Orthogonal Mode, ASME J. of Heat Transfer, 116(2), $369-380$.

Thomas, J. L., Krist, S. T. and Anderson, W. K. (1990) Navier-Stokes Computations of Vortical Flows over LowAspect-Ratio Wings, AIAA J., 28(2), 205-212.

Tolpadi, A. K. (1994) Calculation of Heat Transfer in a Radially Rotating Coolant Passage, AIAA Paper 94-0261.

Tse, D. G. N. (1995) Flow in Rotating Serpentine Coolant Passages with Skewed Trip Strips, Report R95-9089F, Scientific Research Associates, Inc., Glastonbury, Connecticut.

Wagner, J. H., Johnson, B. V. and Hajek, T. J. (1991a) Heat Transfer in Rotating Passages with Smooth Walls and Radia Outward Flow, ASME J. of Turbomachinery, 113(1), 42-51.

Wagner, J. H., Johnson, B. V. and Kopper, F. C. (1991b) Heat Transfer in Rotating Serpentine Passages with Smooth Walls, ASME J. of Turbomachinery, 113(3), 321-330.

Wagner, J. H., Johnson, B. V., Graziani, R. A. and Yeh, F. C (1992) Heat Transfer in Rotating Serpentine Passages with Trips Normal to the Flow, ASME J. of Turbomachinery, 114(4), 847-857.

Wagner, J. H. and Steuber, G. D. (1994) Private Communication.

Wang, T.-S. and Chyu, M. K. (1994) Heat Convection in a $180^{\circ}$ Turning Duct with Different Turn Configurations, AIAA J. of Thermophysics and Heat Transfer, 8(3), 595-601.

Wilcox, D. C. (1993) Turbulence Modeling for CFD, DCW Industries, La Canada, California.

Zhang, N., Chiou, J., Fann, S. and Yang, W.-J. (1993) Local Heat Transfer Distribution in a Rotating Serpentine RibRoughened Flow Passage, ASME J. of Heat Transfer, 115(3), $560-567$.

Zhang, Y. M., Han, J. C., Parsons, J. A. and Lee, C. P. (1995) Surface Heating Effect on Local Heat Transfer in a Rotating Two-Pass Square Channel with $60^{\circ}$ Angled Rib Turbulators, ASME J. of Turbomachinery, 117(2), 272-280. 

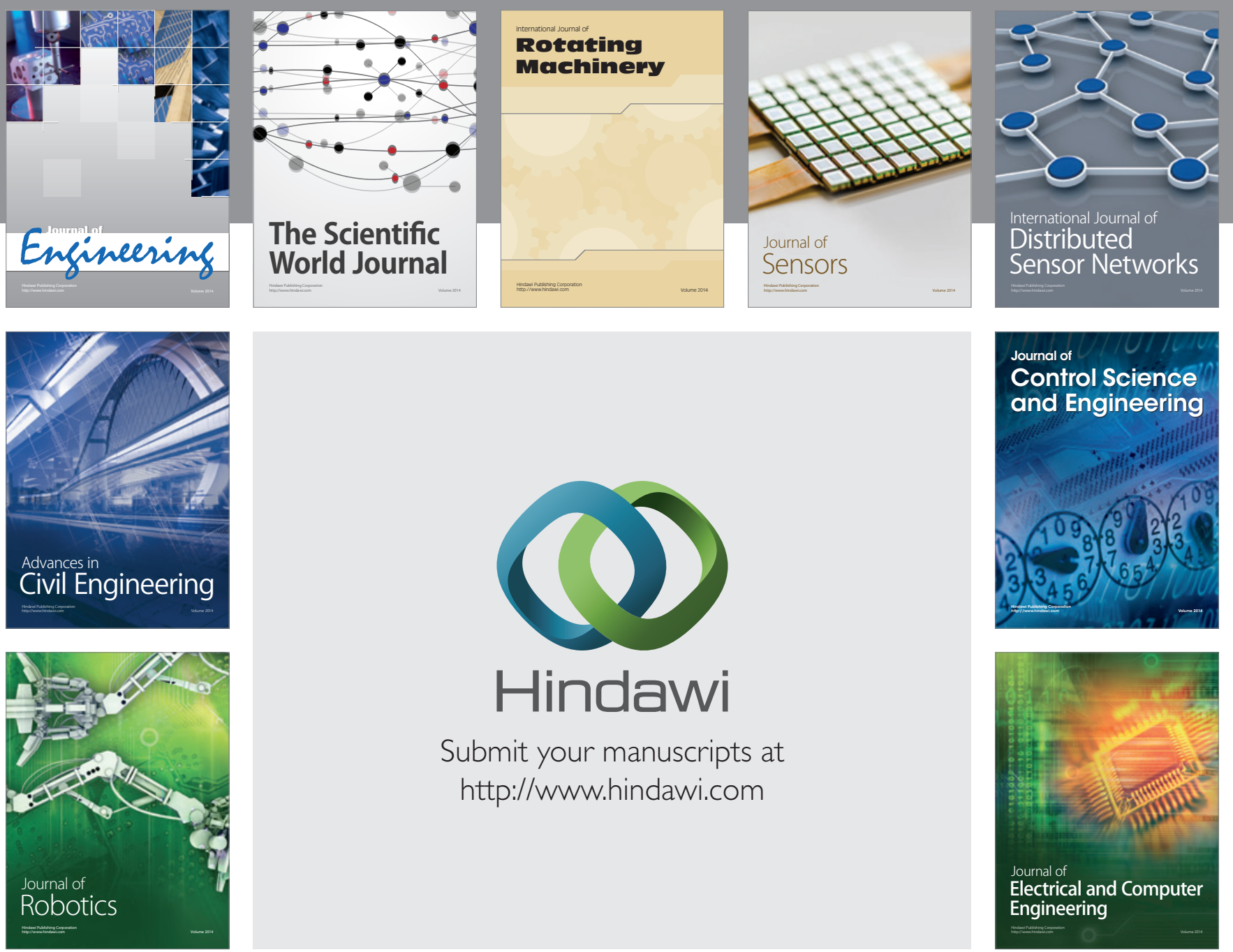

Submit your manuscripts at

http://www.hindawi.com
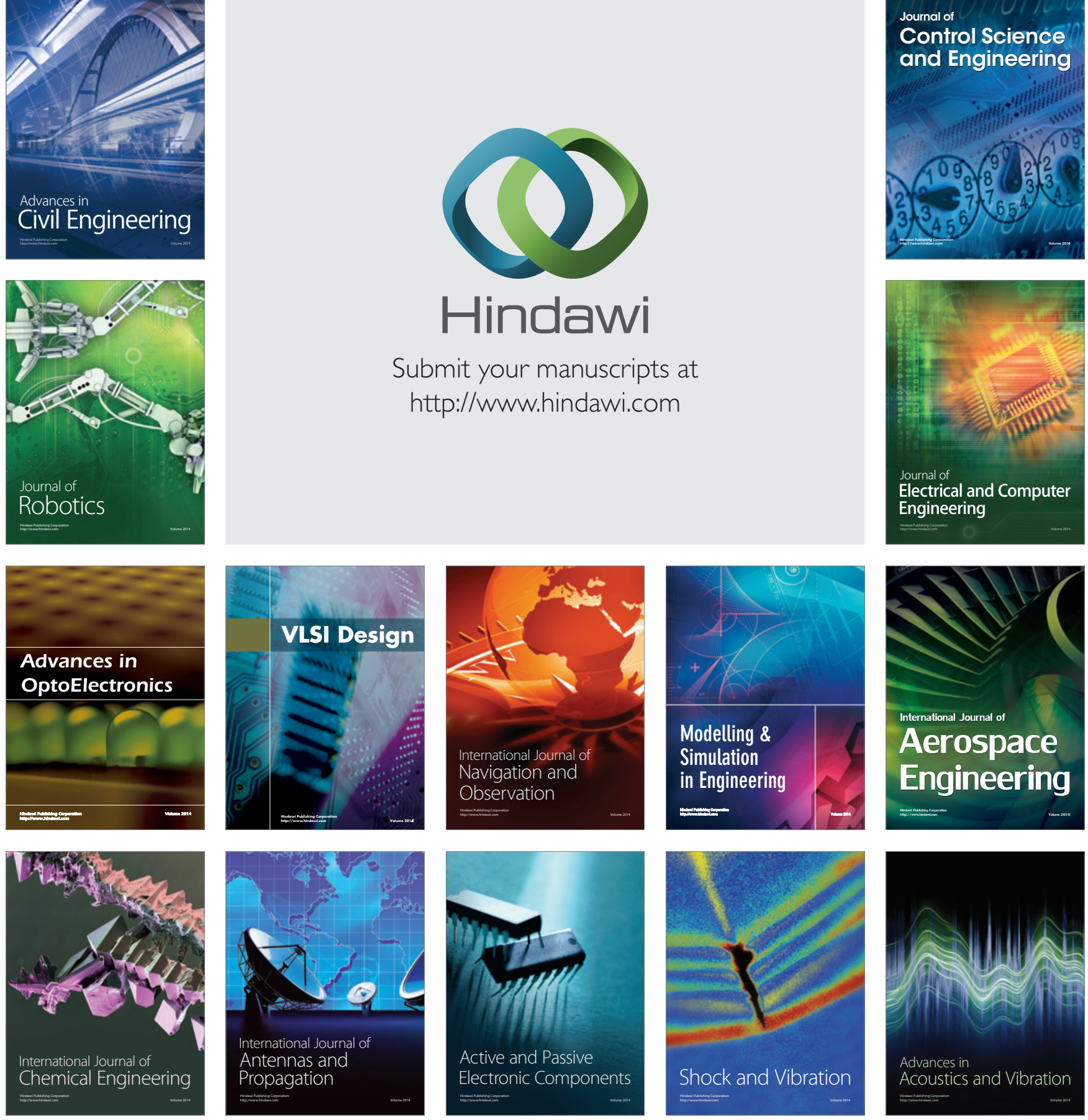